\title{
Satellites Form Fast \& Late: a Population Synthesis for the Galilean Moons
}

\author{
M. Cilibrasi ${ }^{1,2,3 \star}$, J. Szulágyi ${ }^{4,3}$ L. Mayer ${ }^{3}$, J. Dra̧żkowska ${ }^{3}$ and Y. Miguel ${ }^{5,6}$ \\ ${ }^{1}$ Scuola Normale Superiore, Piazza dei Cavalieri 7, I-56126 Pisa, Italy \\ ${ }^{2}$ Dipartimento di Fisica "Enrico Fermi", Università di Pisa, Largo Pontecorvo 3, I-56127 Pisa, Italy \\ ${ }^{3}$ Center for Theoretical Astrophysics and Cosmology, Institute for Computational Science, University of Zürich, \\ Winterthurestrasse 190, CH-8057 Zürich, Switzerland \\ ${ }^{4}$ ETH Zürich, Institute for Particle Physics and Astrophysics, Wolfgang-Pauli-Strasse 27, CH-8093, Zürich, Switzerland \\ ${ }^{5}$ Observatoire de la Côte d'Azur, Boulevard de l'Observatoire, CS 34229 F-06304, Nice Cedex 4, France \\ ${ }^{6}$ Leiden Observatory, University of Leiden, Niels Bohrweg 2, 2333CA Leiden, The Netherlands
}

Accepted XXX. Received YYY; in original form ZZZ

\begin{abstract}
The satellites of Jupiter are thought to form in a circumplanetary disc. Here we address their formation and orbital evolution with a population synthesis approach, by varying the dust-to-gas ratio, the disc dispersal timescale and the dust refilling timescale. The circumplanetary disc initial conditions (density and temperature) are directly drawn from the results of $3 \mathrm{D}$ radiative hydrodynamical simulations. The disc evolution is taken into account within the population synthesis. The satellitesimals were assumed to grow via streaming instability.

We find that the moons form fast, often within $10^{4}$ years, due to the short orbital timescales in the circumplanetary disc. They form in sequence, and many are lost into the planet due to fast type I migration, polluting Jupiter's envelope with typically 0.3 Earth-masses of metals, and up to 10 Earth-masses in some cases. The last generation of moons can form very late in the evolution of the giant planet, when the disc has already lost more than the $99 \%$ of its mass. The late circumplanetary disc is cold enough to sustain water ice, hence not surprisingly the $85 \%$ of the moon population has icy composition. The distribution of the satellite-masses is peaking slightly above Galilean masses, up until a few Earth-masses, in a regime which is observable with the current instrumentation around Jupiter-analog exoplanets orbiting 1 AU away from their host stars. We also find that systems with Galilean-like masses occur in $20 \%$ of the cases and they are more likely when discs have long dispersion timescales and high dust-to-gas ratios.
\end{abstract}

Key words: planets and satellites, formation - planets and satellites, gaseous planets - planets and satellites, general

\section{INTRODUCTION}

In the last few years theories about our Solar System formation took a step forward thanks to a more precise comprehension of giant planet formation and evolution within protoplanetary discs. Today the two main models in this field are the Gravitational Instability scenario, or GI (Boss 1997), when a self-gravitating gaseous clump directly collapses into a giant planet, and the Core Accretion model, or CA (Pollack et al. 1996), that occurs when collisions and coagulation of dust particles form a solid planetary embryo, massive enough to accrete and maintain a gaseous envelope. Both of these theories predict the presence of circumplanetary discs (CPDs) made of gas and dust rotating around the forming planet in the last stage of formation (Alibert et al. 2005; Ayliffe \& Bate 2009; Ward \& Canup 2010; Szulágyi et al. 2017a). Even though these discs are similar to protoplanetary discs (PPDs) around young stars, there are significant differences among them. The most important one is that the CPDs are continuously fed by a vertical influx of gas and well coupled dust from the protoplanetary disc upper layers, due to gas accretion onto the central giant planet (Tanigawa et al. 2012; Szulágyi et al. 2014).

Due to the fact that regular satellites (including the

^ E-mail: marco.cilibrasi@sns.it 
moons of Jupiter) most commonly form in CPDs, the understanding of the properties of these discs is crucial to address satellite formation. With no observational constraints about them, so far we have to rely on hydrodynamic simulations to study the initial CPD that have formed the Galilean satellites (e.g. Ayliffe \& Bate 2009; Gressel et al. 2013; Szulágyi et al. 2017b). The properties of Jupiter's four biggest moons, however, provide some constraints about the features of this disc. Voyager and Galileo missions revealed that Io is rocky, while the outer three moons contain significant amount of water ice (Showman \& Malhotra 1999). The accretion of icy satelletesimals is only possible in a CPD which has a bulk temperature below the water freezing point, $\sim 180 \mathrm{~K}$. However, hydrodynamic simulations of CPDs found the temperature to be significantly higher than that, often peaking at several thousands of Kelvins (e.g. Ayliffe \& Bate 2009; Szulágyi et al. 2016). The study of Szulágyi (2017) showed that even accounting for the cooling of the planet (due to radiating away its formation heat), the Jupiter surface temperature had to be significantly lower than $1000 \mathrm{~K}$, when the Galilean satellites have formed, otherwise the CPD cannot form icy satellites. This indicated that the moons had to form very late in the planet- \& disc-evolution, when Jupiter has significantly cooled off and its CPD was dissipating (moving towards the optically thin, and hence cold regime).

Regarding the mass of the CPD, we know that the total mass of the Galilean satellites is $\sim 2 \times 10^{-4} M_{\text {planet }}$ $\left(M_{p}\right.$ hereafter), same as in the case of Saturn (Canup \& Ward 2006). Because this value considers only solids, with a standard dust-to-gas ratio of 0.01 one gets a CPD mass of $\sim 2 \times 10^{-2} M_{p}$. However, as the Canup \& Ward works have pointed out (Canup \& Ward 2002, 2006, 2009), this is the integrated CPD mass, i.e. at a snapshot of time the CPD can be much lighter than this while still producing Galilean mass satellites over the years (gas-starved disc model). Due to the continuous feeding from the protoplanetary disc, throughout the lifetime of the CPD, even orders of magnitude more material could have been processed through the CPD. The mass of the disc has been certainly enough to make several generations of Galilean-mass moons, and several of them could have been lost into the planet through migration, opening the idea of sequential satellite-formation (Canup \& Ward 2002).

There have been several different approaches to study satellite formation, starting from works that studied conditions of the CPD during satellite formation and constraints on this disc based on the properties of the Galilean moons (Canup \& Ward 2009, 2002; Estrada et al. 2009). Recently, Fujii et al. (2017) numerically solved a 1D-model of circumplanetary disc long term evolution and the migration of satellites in it. They found that the moonlets are often captured in resonances, which could explain the formation of the first three resonant satellites. A population synthesis work made by Sasaki et al. (2010) modeled the initial circumplanetary disc density profile solving a 1D equation for its viscous evolution (Pringle 1981) with an inner cavity between the planet and disc. They included satellite accretion with gravitational focusing and the type I migration timescale using the formula from Tanaka et al. 2002. Building a semi-analytical model and performing a population synthesis varying the location of the initial seeds, the $\alpha$ viscosity and the dispersion time of the disc, they found that in $70 \%$ of their runs they had 4 or 5 satellites, often locked in a resonant configuration thanks to the inner cavity of the disc. They varied the initial circumplanetary disc profiles and used quite different models than what recent hydrodynamic models on the CPD predict (e.g. Ayliffe \& Bate 2009, Tanigawa et al. 2012, Szulágyi et al. 2014, Szulágyi 2017). Same is true for the Miguel \& Ida (2016), which used the Minimum Mass Subnebula (Mosqueira \& Estrada 2003) as an initial CPD profile. They studied the evolution of about 20 satellite-seeds, with initial positions randomly chosen in the disc, together with the gas density of the disc (but without the temperature evolution in their case), considering also the dust depletion caused by the accretion of dust itself onto protosatellites. Different runs have been made with different disc parameters, such as the dust-to-gas ratio of the disc, its dispersion timescale and the initial mass of satellitesimals, using then a population synthesis approach to analyze the outcomes.

0Because previous works have used CPD profiles that were derived from the current composition and location of the Galilean satellites without taking into account their migration and the possibility for several lost satellites system, here we present a population synthesis on CPD profiles that are consistent with recent radiative hydrodynamical simulations on the circum-Jovian disc. We also take into account the thermal evolution of the disc, and the continuous feeding of gas and dust from the vertical influx from the protoplanetary disc (e.g. Tanigawa et al. 2012; Szulágyi et al. 2014; Fung \& Chiang 2016). Moreover, we use a dust-coagulation and evolution code to calculate the initial dust density profile corresponding to the gas hydrodynamics of Szulágyi (2017). We assumed that the initial seeds were formed via streaming instability (e.g. Youdin \& Goodman 2005), and we placed these moonlets at the location where the conditions for streaming instability are satisfied (e.g. the local dust-to-gas ratio is higher than unity).

\section{METHODS}

\subsection{Hydrodynamic simulation}

For the circumplanetary disc density and temperature profiles we used a simulation from Szulágyi (2017). Among the various models in that paper considering different planetary temperatures, we used here one of the coldest (most evolved) state with planetary temperature of $2000 \mathrm{~K}$. This is because the satellites of Jupiter are icy, they had to form in a cold circumplanetary disc, when the planet has cooled off efficiently (Szulágyi 2017). This is only true in the very late stage of circumplanetary disc evolution, close to the time when the circumstellar disc has dissipated away.

Our hydrodynamic simulation was performed with the JUPITER hydrodynamic code (de Val-Borro et al. 2006; Szulágyi et al. 2016) developed by F. Masset \& J. Szulágyi. This code is three dimensional, grid-based, uses the finitevolume method and solves the Euler equations, the total energy equation and the radiative transfer with flux limited diffusion approximation, according to the two-temperature approach (e.g. Kley 1989; Commerçon et al. 2011). The simulation contained a circumstellar disc between 2.08 AU till 12.40 AU (sampled in 215 cells radially), with an initial 
opening angle of 7.4 degrees (from the midplane to the disc surface, using 20 cells). The coordinate system in the simulation was spherical, centered on the Sun-like star and corotating with the planet. The initial surface density was a power-law function with $2222 \mathrm{kgm}^{-2}$ at the planet's location at 5.2 AU and an exponent of -0.5. The planet was a Jupiter analog, which reached its final mass through 30 orbits. The circumstellar disc azimuthally ranged over $2 \pi$ sampled into 680 cells. To have sufficient resolution on the circumplanetary disc developed around the gas-giant, we placed 6 nested meshes around the planet, each doubling the resolution in each spatial direction. Therefore, on the highest resolution mesh the sampling was $\sim 80 \%$ of Jupiter-diameter $(\sim 112000$ $\mathrm{km}$ ) for a cell-diagonal. For the boundaries and resolution of each refined level, we used the same as Table 1 in Szulágyi et al. (2016). Because the resolution is sub-planet resolution, at the planet location we fixed the temperature to $2000 \mathrm{~K}$ (thereafter referred as planet temperature) within $3 \mathrm{R}_{\text {Jupiter }}$, corresponding an evolved, late stage of the circumstellar disc and planet system, roughly around 1-2 Myrs.

The equation of state in the simulation was ideal gas $P=(\gamma-1) \mathrm{E}_{\text {int }}-$ which connects the internal energy $\left(\mathrm{E}_{\mathrm{int}}\right)$ with the pressure (P) through the adiabatic exponent: $\gamma=1.43$. For the viscosity, we solve the viscous stress tensor to set a constant, kinematic (physical) viscosity, that equals to 0.004 $\alpha$-viscosity at the planet location. Due to the radiative module and the energy equation, the gas can heat up through viscous heating, adiabatic compression and cool through radiation and adiabatic expansion. The opacity table used in the code was of Bell \& Lin (1994) that contains both the gas and dust Rosseland-mean opacities. Therefore, even though there is no dust component explicitly included into the simulations, the dust contribution to the temperature is taken into account through the dust-to-gas ratio, that was chosen to be 0.01 , i.e. equal to the interstellar medium value (Boulanger et al. 2000). The mean-molecular weight was set to 2.3 , which corresponds to solar composition. The rest of the parameters and process of the simulation can be found in Szulágyi (2017) and Szulágyi et al. (2016).

\subsection{Population synthesis}

Our semi-analytical model essentially consist of a circumplanetary disc in which protosatellites can migrate, accrete mass and be lost into the central planet. In the meantime, while the disc density and temperature evolve in time, it creates newer and newer protosatellites. The units in our population synthesis are the following: $R_{p}$ as planet radius, $M_{p}$ (planetary mass), time in years and temperature in Kelvin.

\subsubsection{Disc structure}

In the model, the CPD is simply defined by its surface density (both solid and gas) profiles, temperature profile and other quantities, such as $\alpha$ for viscosity, $\gamma$ for heat capacity ratio and $C_{V}$ for heat capacity at constant volume. All other quantities in the disc, such as the angular velocity of the gas, the height of the disc, the speed of sound, etc., are computed starting from temperature and density values and using the common 1D model for discs (Pringle 1981). The disc ranges between $1 R_{p}$ and $500 R_{p}$, according to the hydrodynamical simulation, and it is divided in 500 cells. In our model we do not consider a cavity between the planet's surface and the disc, because the magnetic field of the planet and the ionization of the disc are probably not strong enough to produce such a cavity (see also in Section 4). The disc initial temperature and gas density profiles are power-law fits to the results of a radiative hydrodynamical simulation of Szulágyi (2017) with planet temperature of $2000 \mathrm{~K}$ (i.e. a late time in the evolution of the forming planet \& its disc, corresponding to roughly 1-2 Myrs of PPD age), described in section 2.1. The power-laws are the followings (Figure 1, Figure 2):

$$
\begin{aligned}
& \Sigma_{\text {gas }}(r) \simeq 4.8 \cdot 10^{-6}\left(\frac{r}{R_{p}}\right)^{-1.4}\left[\frac{M_{p}}{R_{p}^{2}}\right] \\
& T(r)= \begin{cases}1.4 \cdot 10^{4}\left(\frac{r}{R_{p}}\right)^{-0.6}[K] & T_{\text {min }}<T<T_{\text {max }} \\
T_{\text {min }} & T \leq T_{\text {min }} \\
T_{\text {max }} & T \geq T_{\text {max }}\end{cases}
\end{aligned}
$$

with $T_{\text {min }}=130 \mathrm{~K}$, that is the background temperature in the PPD at Jupiter's location like e.g. in Miguel \& Ida (2016), and $T_{\max }=2000 \mathrm{~K}$, that is the planet temperature in the simulations. The total disc mass is $M_{0} \simeq 2 \times 10^{-3} M_{p}$, always accordingly to the $3 \mathrm{D}$ hydrodynamic simulation. Other parameters are chosen to be consistent with the hydrodynamic simulation, therefore the viscosity is $\alpha=0.004$, the adiabatic index is $\gamma=7 / 5$ (i.e. molecular hydrogen) and the heat capacity $\left(C_{V}\right)$ equals to $10.16 K J /(K g K)$, again because of consistency with the hydro simulation.

Because the hydrodynamical simulation only gives the gas density profile, we used the dust density profile of Drazkowska \& Szulagyi (in prep), where dust coagulation and evolution is computed on the same gas density profiles (Figure 1). As the figure shows, there is a peak in the dust density at around $85 R_{J}$. This dust trap is the consequence of the position where the radial velocity of gas changes sign in the hydro simulation, i.e. the gas is bringing small dust particles from the inner and outer disc to this location. The temperature of the dust was assumed to be the same as the gas temperature, assuming perfect thermal equilibrium.

Given that the dust-to-gas ratio of the CPD is not known, we kept it as a free parameter in the population synthesis. Therefore the dust density profiles where multiplied by a scalar in each individual run of the population synthesis. This is not exactly accurate since simulations do not show a simple linear scaling, but we checked that this assumption does not change results significantly. In Figure 1 the dust-to-gas ratio at the equilibrium is 0.08 . In this work we always refer to this final ratio, however Drazkowska \& Szulagyi (in prep) found that this final and equilibrium value is about 5.8 times the initial dust-to-gas ratio of the simulated disc.

In our model, we do not consider the jump of the solid density beyond the ice line, that could happen due to the condensation of water (see for example Mosqueira \& Estrada 2003; Miguel \& Ida 2016; Drazkowska \& Alibert 2017). In fact, the code of Drazkowska $\&$ Szulagyi (in prep) found that the dust dynamics is dominated by the gas flow and thus, even after the ice line enters the disc because of cooling, the modification to the dust surface density (in Figure 1) due to the existence of solid ice is negligible, it only affects the 


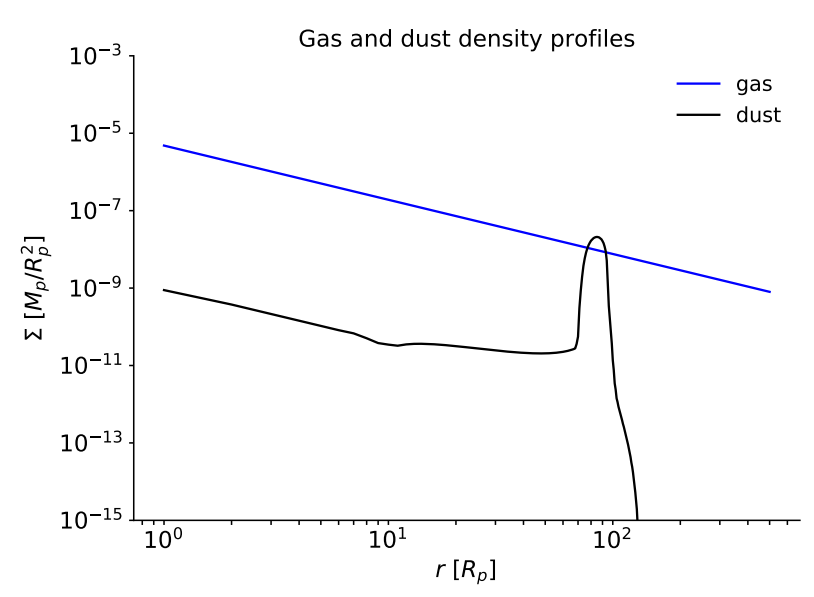

Figure 1. Gas (blue) and dust (black) density profiles of the circumplanetary disc at the beginning of the population synthesis. The dust-to-gas ratio here was chosen to be 0.08 , but this parameter is varied in the population synthesis.

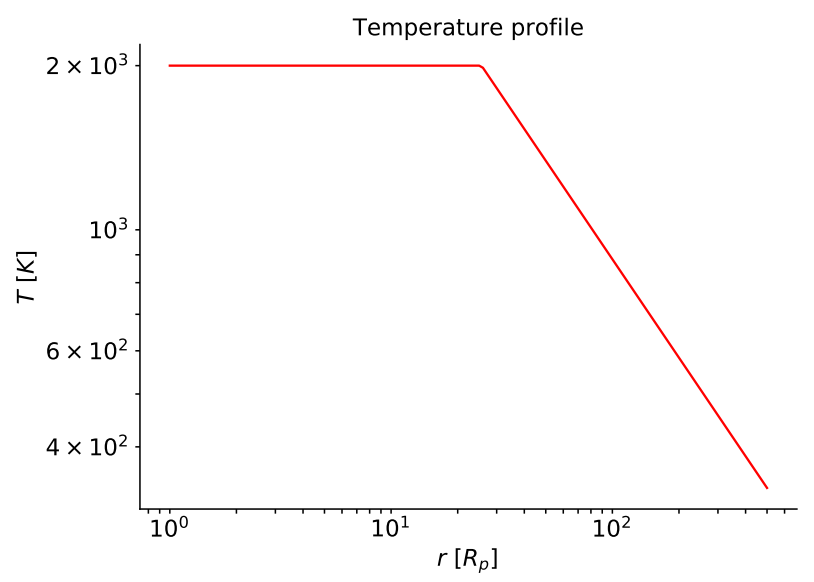

Figure 2. Temperature profile of the disc at the beginning of the population synthesis. $T_{\max }=2000 \mathrm{~K}$ corresponds to the planet temperature, while $T_{\min }=130 \mathrm{~K}$ is the background temperature at Jupiter's location (5.2 AU from the Sun).

composition of the dust profile in terms of volatiles. Since we are not interested in the exact composition of the dust (see Section 3.5 for more details) we simply neglected the possibility to have a dust density-jump at the ice line.

\subsubsection{Disc evolution}

We adopted a self-similar solution for disc evolution. It is known that the disc is fed by the vertical influx from the protoplanetary disc (Tanigawa et al. 2012; Szulágyi et al. 2014) that should decrease exponentially with time as

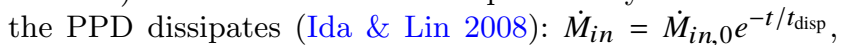
where $t_{\text {disp }}$ is the characteristic dispersion time of PPD and $\dot{M}_{i n, 0} \simeq 2 \times 10^{-6} M_{p} / y r$ in our case, in agreement with numerical simulations in Section 2.1. The mass loss is assumed to be proportional to the mass of the disc itself: $\dot{M}_{\text {out }}=\dot{M}_{\text {out }, 0} \frac{M}{M_{0}}$.
We also assume that the CPD is initially at the equilibrium, i.e. $\dot{M}_{i n, 0}=\dot{M}_{\text {out }, 0}=\dot{M}_{0}$. Solving the equation

$\frac{d M}{d t}=\dot{M}_{\text {in }}-\dot{M}_{\text {out }}$

one can find that if $t_{\text {disp }} \gg M_{0} / \dot{M}_{0}$, and it is always the case in our model (see the values for $t_{\text {disp }}$ in the next paragraph), the CPD density decreases exponentially with $t_{\text {disp }}$ keeping the equilibrium configuration, following in practice the decrease of $\dot{M}_{i n}(t)$. Therefore, in our population synthesis, the disc density evolution is given by:

$\left\{\begin{array}{l}\Sigma_{g}=\Sigma_{g, 0} e^{-t / t_{\mathrm{disp}}} \\ \Sigma_{s}=\Sigma_{s, 0} e^{-t / t_{\mathrm{disp}}}-A\end{array}\right.$

where $t_{\text {disp }}$ is the dispersion time of the CPD (that is equal to the dispersion time of the PPD), while $A$ is the dust accreted by the protosatellites and then regenerated by the refilling mechanism, as it will be explained in section 2.2.3.

The disc dispersion timescale and the total disc lifetime are not the same thing but they are not independent from each other as well, hence we also linked them in our calculation. Recent observations showed that disc lifetimes distribute exponentially between $1 M y r$ and $10 M y r$ with a characteristic age of 2.3Myr (Fedele et al. 2010; Mamajek 2009). These surveys have an accreation rate sensitivity limit till $>10^{-11} M_{\odot} y r^{-1}$, however, on average, young $\mathrm{T}$ Tauri star with a protoplanetary disc shows an accretion rate of $\sim 10^{-7} M_{\odot} y r^{-1}$ (e.g. Ercolano et al. 2014). Considering these limits, and considering the exponential evolution of disc density (and mass), the disc lifetime will be:

$t_{\text {lifetime }}=-t_{\text {disp }} \ln \left(\frac{10^{-11} M_{\odot} y r^{-1}}{10^{-7} M_{\odot} y r^{-1}}\right) \simeq 10 t_{\text {disp }}$

where the dispersion timescales are distributed exponentially between $0.1 M y r$ and $1.0 M y r$, with a mean of $0.23 M y r$.

The temperature evolution was calculated also with an exponential decrease to be consistent with the density evolution:

$T=T_{\text {min }}+\left(T_{0}-T_{\text {min }}\right) e^{-t / t_{\text {cool }}}$

where $t_{\text {cool }}$ is computed with the radiative cooling formula of Wilkins \& Clarke (2012):

$\dot{T} \propto \dot{U}=-\sigma \frac{T^{4}-T_{\text {min }}^{4}}{\Sigma_{g}\left(\tau+\tau^{-1}\right)}$

The optical depth $(\tau)$ can be estimated as $\tau=\int \rho \kappa d h \simeq \kappa \Sigma_{g}$, where $\kappa(\Sigma, T)$ is the opacity computed with tables in Zhu et al. (2009).

As the optical depth $(\tau)$ depends only on $T$ and $\Sigma$, therefore the cooling depends only on how $\Sigma$ varies with time, and it is possible to find a relation between $t_{\text {cool }}$ and $t_{\text {disp }}$ (Fig. $3)$. Defining $t_{\text {cool }}$ as the time at which the total internal energy of the disc divided by the total mass of the disc itself $(T \propto U / M)$ is $1 / e$ of its initial value, as it can be seen in Figure 4, where it is also clear the exponential nature of the cooling process. This relation is found by fitting the results with $t_{\text {disp }}$ between $10^{5} y r$ and $10^{6} y r$ :

$\log _{10}\left(t_{\text {cool }}\right)=-0.11 \log _{10}\left(t_{\text {disp }}\right)^{2}+1.9 \log _{10}\left(t_{\text {disp }}\right)-1.5$

where timescales are in years. We also show this fit in Figure 3. 


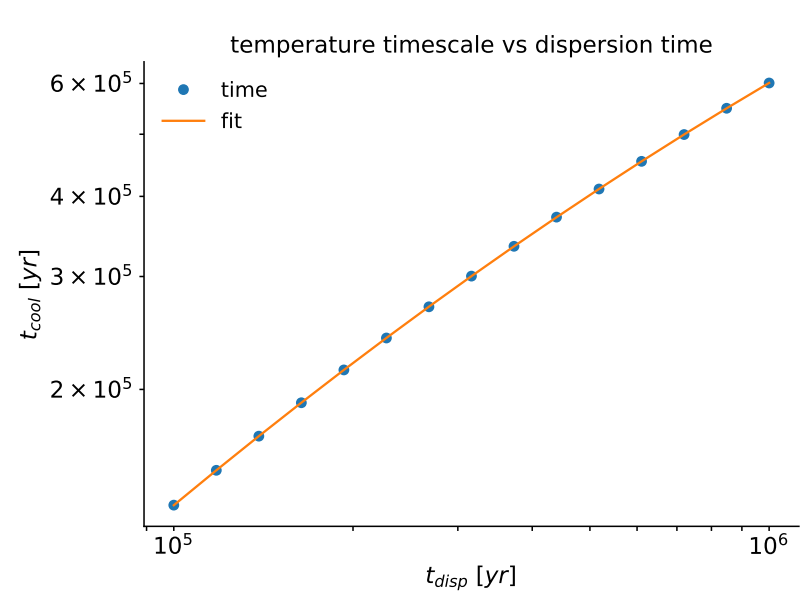

Figure 3. Relation between $t_{\text {cool }}$ and $t_{\text {disp. The blue dots are the }}$ result for 15 different values of $t_{\text {disp }}$ while the orange line is the fit given by equation 8 .

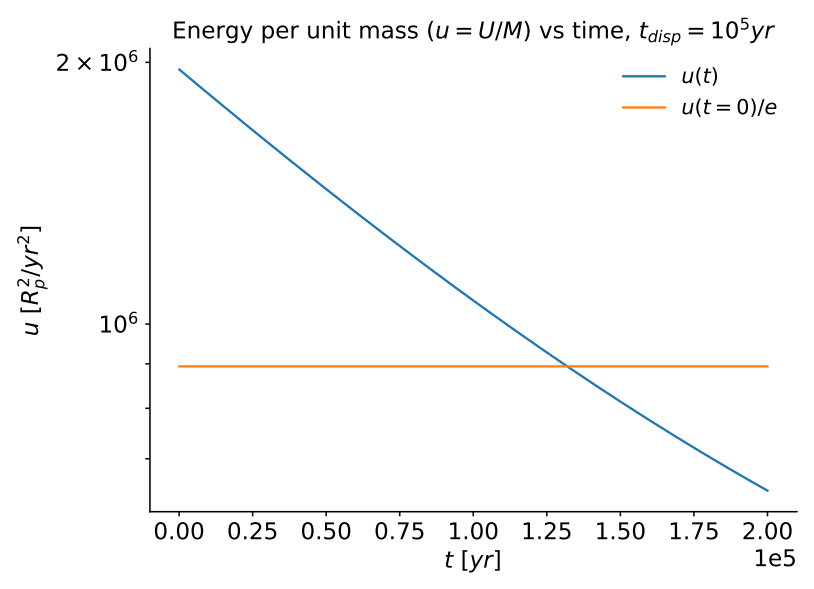

Figure 4. Energy per unit mass $u$ in case of $t_{\text {disp }}=10^{5} y r$. The orange line present the initial value for energy divided by $e$ and the blue line is the energy evolution (cooling). The cooling-curve (blue) is nearly exponential.

\subsubsection{Protosatellite formation and evolution}

Satellite Formation and Loss Once a simulation has started, the code starts to create a new embryo in the position of the dust trap, assuming that the mechanism for dust coagulation is streaming instability (Youdin \& Goodman 2005 ), i.e. a mechanism in which the drag felt by solid particles orbiting in a gas disc leads to their spontaneous concentration into clumps which can gravitationally collapse. The moonlet formation process starts when these two conditions occur:

(i) The ratio between the solid density and the gas density in the midplane of the dust trap is more than 1 . This condition can occur only if the global dust-to-gas ratio is high enough ( $\geq 0.03$ is the threshold in the model, i.e. the initial dust-to-gas ratio should be $\geq 0.005)$. This value is given by the profile definition in section 2.2.1. (ii) The previous proto-moon is far enough, i.e. the dust trap is out of its feeding zone, because of migration.

Once these two conditions has occurred the embryo has to grow to the fixed initial mass $\left(m_{0}=10^{-7} M_{p}\right.$, that is more than two orders of magnitude smaller than individual masses of the Galilean satellites). We use also a formation rate $\left(\dot{m}_{0}\right)$ taken from Drazkowska \& Szulagyi (in prep), which we assume to decrease at the same rate as the circumplanetary disc density decreases, i.e. $\dot{m}=\dot{m}_{0} e^{-t / \tau}$. Starting from the moment in which the two above-mentioned conditions occur we integrate this formation rate in time until $m=m_{0}$. At this point the code creates the new protosatellite in the disc. The value for $m_{0}$ is arbitrary and we tested various $m_{0}$ to make sure that this initial parameter does not affect results.

The evolution of a protosatellite is stopped in two occasions:

(i) When a protosatellite reaches the inner boundaries of the disc, then the satellite is considered to be lost into the planet.

(ii) When two protosatellites intersect their paths the code stops the smallest of the two. (We are neglecting the possibility that 2 satellites pass each other in 3D.)

The simulation ends when the total lifetime of the disc is reached, i.e. when $\frac{t}{t_{\text {disp }}} \sim 10$ (see in Section 2.2.2).

Migration In the code we distinguish between type I migration and type II migration. Gap opening separates the two regimes, therefore we use the gap opening parameter $P=\frac{3}{4} \frac{h}{R_{H}}+\frac{50}{q R e}=\frac{3}{4} \frac{c_{s}}{\Omega_{K} a}\left(\frac{M_{s}}{3 M_{p}}\right)^{-1 / 3}+50 \alpha \frac{M_{p}}{M_{s}}\left(\frac{c_{s}}{\Omega_{K} a}\right)^{2}$ from Crida \& Morbidelli (2007). We consider that type I takes place if $P>1$, otherwise (if $P<1$ ) type II operates.

To compute type I migration velocity we use

$v_{r}=b_{I} \frac{M_{s} \Sigma_{g} a^{3}}{M_{p}^{2}}\left(\frac{a}{h}\right)^{2} \Omega_{K}$

where $M_{S}$ is the mass of the satellite and $b_{I}$ is a parameter that is widely used in the migration community and has been computed in different disc conditions (Paardekooper et al. 2011; D'Angelo \& Lubow 2010; Dittkrist et al. 2014). In our code we use the $b_{I}$ obtained in 3D non-isothermal simulations in Paardekooper et al. (2011), as a function of the disc density, temperature and satellite mass. One has also to consider the fact that when a satellite is growing, it is also starting to open a partial gap, therefore the gas density is decreasing in the closer Lindblad locations and as a consequence, migration velocity decreases. This is done by multiplying $b_{I}$ by the value of the gap depth $(0 \leq$ depth $\leq 1)$ according to the analytic formula of Duffell (2015).

In type II migration, the satellite migrates with the gap, with velocity computed as in Pringle (1981):

$v_{r}=-3\left(\beta_{\Sigma}+\beta_{T}+2\right) \frac{\alpha c_{s} h}{a}$

where $\beta_{\Sigma}=-\frac{d \ln \Sigma_{g}}{d \ln r}$ and $\beta_{T}=-\frac{d \ln T}{d \ln r}$, or $v_{r}=-\frac{3}{2} \frac{\alpha c_{s} h}{a}$ in steady state discs, from which it is possible to define a second $b$ parameter, i.e. $b_{I I}=-\frac{3}{2} \frac{c_{s}^{4} \alpha}{\Omega_{K}^{4} a^{6} \Sigma_{g} M_{s}}$. We also want to underline that $b_{I I}$ becomes smaller by a factor of $\sim M_{S} /\left(4 \pi a^{2} \Sigma_{g}\right)$ when the satellite grows in mass (Syer \& Clarke 1995) and 
changes migration regime (from disc-dominated to satellitedominated). So we modify $b_{I I}$ as:

$$
b_{I I} \rightarrow \frac{b_{I I}}{1+\frac{M_{s}}{B}} \quad, \quad B=4 \pi a^{2} \Sigma_{g}
$$

Furthermore, we also considered a smooth transition between type I and type II migration by using a junction function $z$ from Dittkrist et al. (2014):

$b=z(1 / P) b_{I}+[1-z(1 / P)] b_{I I}$

where $z(x)=\frac{1}{1+x^{30}}$ and $P$ is the gap opening parameter.

Since the Galilean satellites are found in resonances, we also tried to resonant trapping in our population synthesis. Actually, the resonance capturing turned out to be a very rare phenomenon in our model because the inner satellite has to slow down significantly for capture to occur, because it is necessary to have converging orbits. The only strong slowing mechanism in our model would be the gap opening, but as we show in Section 3.3, this happens very rarely.

The migration rates are also used to compute the time-steps in the code. In more detail, the time-steps are never longer than $t_{\text {disp }} / 100$ in order not to lose precision on the disc evolution. Moreover, we also impose that a satellite should never move for more than one tenth of a disc cell (i.e. $1 R_{p} / 10$ ) during its migration. As a consequence each timestep is the minimum value between $t_{\text {disp }} / 100$ and $0.1 R_{p} /\left|v_{\text {mig }}\right|$, computed separately for each migrating satellite.

Accretion While a protosatellite is migrating in the CPD, it also accretes mass from the dust disc. For a very thin dust disc this accretion prescription is (Greenberg et al. 1991):

$\dot{M}_{S}=2 R_{S} \bar{\Sigma}_{s} \sqrt{\frac{G M_{s}}{R_{S} v_{K}^{2}}} v_{K}=2\left(\frac{R_{S}}{a}\right)^{1 / 2} \bar{\Sigma}_{s} a^{2}\left(\frac{M_{s}}{M_{p}}\right)^{1 / 2} \Omega_{K}$

where $R_{S}$ is the radius of the satellite and $\Omega_{K}$ is the Keplerian angular velocity at satellite's position. In the formula, we use $\bar{\Sigma}_{s}$ (that is different from $\Sigma_{s}$ ), because it is the average solid density over the entire feeding zone. The radius of the feeding zone is the same order of magnitude as the Hillradius, i.e. $R_{f}=2.3 R_{H}$ (Greenberg et al. 1991). This value is then multiplied by the gap depth because if the dust is well coupled with the gas (i.e. it is composed by small, $\leq$ $\mathrm{mm}$, grains), then as the satellite grows and opening a gap, there will be less dust around it to accrete.

Once a satellite has accreted the computed mass during a time-step, it is necessary to subtract this mass from the dust disc density. This dust is taken from the feeding zone proportionally to the available mass in each cell: in each point $i$ of the grid within $R_{f}$ solid density decreases by a value of $\Delta M(i)=\frac{M_{\max }(i)}{\sum_{i}^{R_{f}} M_{\max }(i)} d M$ where $M_{\max }(i)$ is the mass available in the $i$-th cell. It often happens that a moonlet accretes all the mass available in the feeding zone, reaching its isolation mass.

After a protosatellite has accreted the mass in the feeding zone and created a gap in the dust, the disc tends to use the dust falling from the PPD's vertical influx to reach the equilibrium again, according to Drazkowska \&s Szulagyi (in prep). But it is also taken account that some of the dust is lost into the central planet, assuming the accretion rate is equal to the dust infall rate. In the population synthesis, we model this refilling mechanism assuming a typical timescale

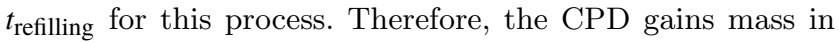
the following way:

$\Delta \Sigma_{s}= \begin{cases}\frac{\hat{\Sigma}_{s}-\Sigma_{s}}{t_{\text {refilling }}} d t & d t \leq t_{\text {refilling }} \\ \hat{\Sigma}_{s}-\Sigma_{s} & d t>t_{\text {refilling }}\end{cases}$

where $d t$ stands for the time-step, $\Sigma_{s}$ is the current solid density and $\hat{\Sigma}_{s}$ is the value that the solid density would have if there was not accretion and consequent depletion.

The timescale of this process is not well constrained, because it strongly depends, for instance, on the amount of dust that fall into the CPD from the PPD, that can be either very fast, with $t_{\text {refilling }} \sim 10^{2} y r$, or very slow, with $t_{\text {refilling }} \sim 10^{6} y r$.

\subsubsection{Population synthesis}

The last module of the code allows to run the semi-analytical algorithm with a population synthesis approach. The idea of population synthesis is to explore a range of the unconstrained parameters, trying all the different combinations between them and in the end to compare the results, individually or grouped. The parameters we vary in the population synthesis are:

- the dust-to-gas ratio in $(0.03,0.50)$, changing only the dust component

- the CPD dispersion timescale: $t_{\text {disp }}$ in $\left(10^{5}, 10^{6}\right) y r$

- the dust refilling timescale: $t_{\text {refilling }}$ in $\left(10^{2}, 10^{6}\right) y r$

In random cases we distribute $t_{\text {disp }}$ exponentially, as described by Fedele et al. (2010), while we distribute dust-togas ratio and $t_{\text {refilling }}$ logarithmically. Furthermore, we vary when the simulation begins, in order to have different initial conditions in temperature and density profiles of the disc. The simulation can start anytime between 0 and $t_{\text {disp }} / 2$.

In principle one can set lower dust-to-gas ratios but since streaming instability is only occurring when the dustto-gas ratio is $>0.03$ we did not consider those low dust-togas ratio cases in our results. There will be, of courses, cases with dust-to-gas ratios $<0.03$ but estimating their number would be possible only when the global dust-to-gas ratio distribution will be clear. For instance, calling the dust-togas ratio variable $x$, if we assume a logarithmic distribution within $0.01<x<0.50$, i.e. $d P / d x \propto 1 / x$, and we extend the distribution in order to go to 0 for low dust-to-gas ratios (for example $d P / d x \propto 100 x$ in $0<x<0.01$ seems reasonable), we find that about $35 \%$ of the cases have dust-to-gas ratio $<0.03$.

One could also vary other parameters, such as the initial embryos mass or the type I migration formula used. We tested these, but this did not change the results much, therefore we kept them fixed as described in the previous sections. We show in Figure 5 how the results of a single run look, with satellites growing, being lost and migrating within a CPD. We also note that there are parameters we kept fixed to be consistent with the hydrodynamic simulation, but they could have been varied too. 

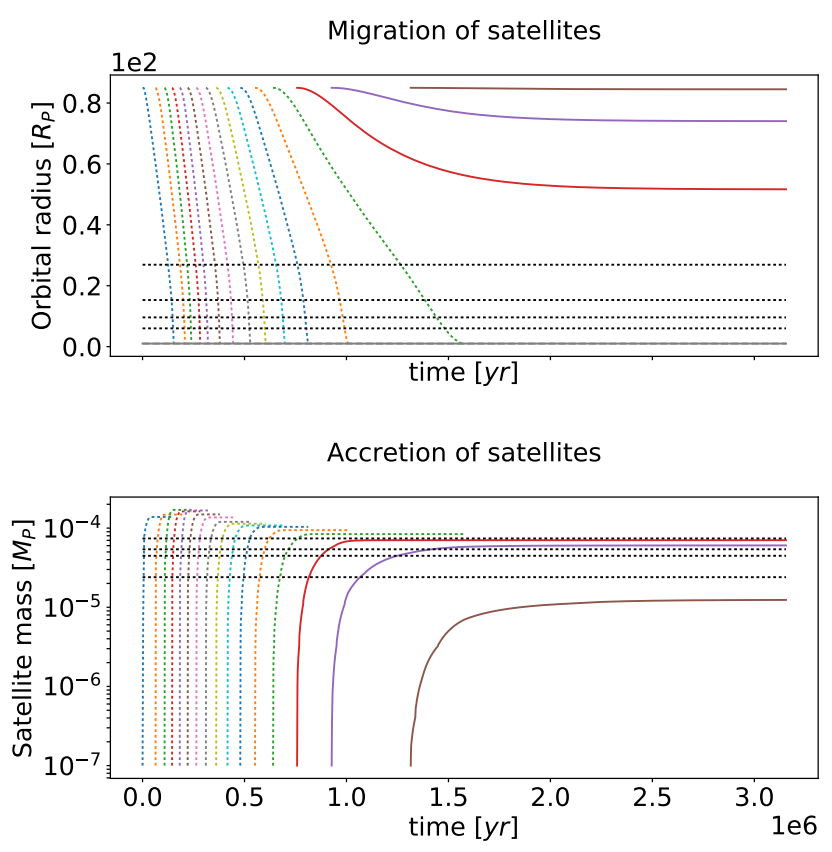

Figure 5. Evolution of satellites in a system with dust-to-gas ratio $=0.1, t_{\text {disp }}=10^{5} \mathrm{yr}$ and $t_{\text {refilling }}=2 \times 10^{4} \mathrm{yr}$. Solid lines are the surviving satellites, dashed lines are lost ones.

\section{RESULTS}

In our work we used two kinds of statistical approaches: the first one consists in running twenty-thousands of different simulations with randomizing the three initial parameters described in the previous section. The second approach is fixing a value for a single parameter, and let the other two vary randomly. The first approach allows to have a general understanding of the outcomes, respecting parameter distribution (especially the exponential distribution of $t_{\text {disp }}$, that is an observational constraint), while the second approach allows to understand how a single parameter affects the results.

\subsection{Survival timescale of the last generation of satellites}

Due to the fact that the moonlets migrate inwards in the disc, and there is no gap between the planet and the CPD, many (even a dozen of) satellites are lost into the planet during disc evolution and therefore only the latest set of moons will survive when the CPD (and PPD) dissipates. This is called sequential satellite formation, that was already suggested in e.g. Canup \& Ward (2002). These lost satellites pollute the envelope of the forming giant planet, increasing the metallicity of the gas-giant. Given that Jupiter's atmosphere is enriched $\sim 2$ times compared to the protosolar values (e.g. Bolton et al. 2017), these lost satellites (and the continuous dust drift/migration) might contribute to this overabundance of heavy elements. Therefore, we computed the mass, what the lost satellites bring into Jupiter: we found a distribution with a median value of $\simeq 10^{-3} M_{J} \simeq 0.3 M_{\oplus}$,

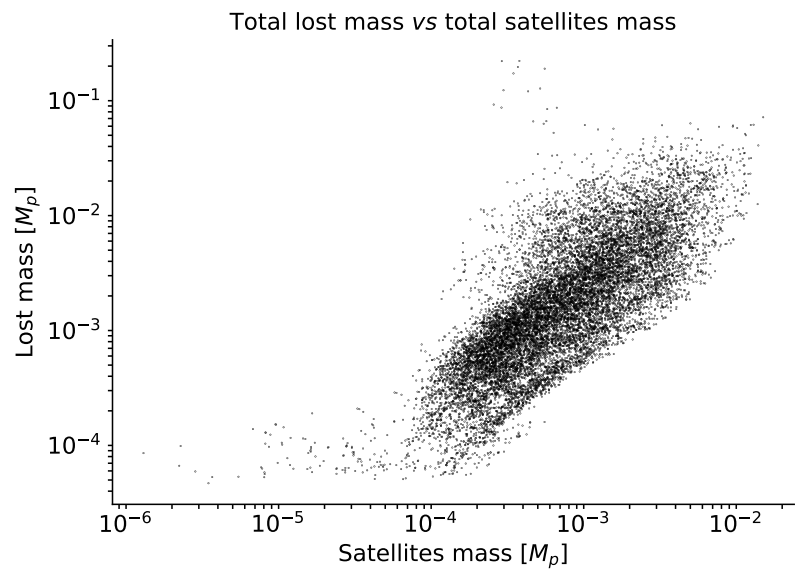

Figure 6. The total lost satellite mass that is polluting the planet versus the total mass of the surviving satellites (see section 3.3). While the median value of lost satellites is only $0.3 M_{\oplus}$, the scatter is large, with values up to $30 \boldsymbol{M}_{\oplus}$ pollution.

but the tail of this distribution covers many cases with a lost mass of $\simeq 10^{-2} M_{J}$ and there are even few cases with a lost mass of $\simeq 0.1 M_{J} \simeq 30 M_{\oplus}$. Nevertheless, in cases in which we have a total mass of satellites similar to the Galilean integrated mass (see Section 3.3) we expect to lose the median value of about $10^{-3} M_{p}$ into the central planet, as shown in Figure 6 .

Proceeding with the first type of population synthesis approach (randomly varying parameters) it is possible to study the general behavior of forming satellite-systems. Running 20000 simulations, we found 4467 (22.34\%) systems in which there are not survived satellites, $325(1.62 \%)$ systems in which all satellites survive and 15208 (76.04\%) systems in which at least one moon is lost but at the same time at least one satellite survives. This fact is confirmed in Figure 7 , where we show the distribution of the time after which the last generation forms (hereafter $t_{L G}$ ) for 20000 satellites. The figure indicates that most of the satellites form between $2 \times 10^{5}$ and $5 \times 10^{6}$ years $(93 \%$ of the cases).

Calculating the distribution of $t_{L G} / t_{\text {disp }}$ allows to study how late satellites form compared to the dispersion timescale of the disc and then, as a consequence, to the total lifetime $\left(t_{\text {life }} \simeq 10 t_{\text {disp }}\right)$. It is clear that they form very late in the system evolution, even after 5 dispersion timescales, i.e. after $50 \%$ of the total lifetime of the disc (see Fig. 8 ), when usually discs are already very poor of gas and dust, having about $0.5 \%$ of the initial mass. Here we always refer to the dispersion timescale because it is the fundamental quantity that defines the evolution of a disc (e.g. $\left.\Sigma(t) \propto \exp \left(-t / t_{\text {disp }}\right)\right)$. Using years, as in Figure 7 , could be misleading, since there is an order of magnitude of difference between the fastest evolving $\operatorname{discs}\left(t_{\text {disp }}=10^{5} y r\right)$ and the slowest one $\left(t_{\text {disp }}=10^{6} y r\right)$.

It is also possible to analyze the model with the second type of population synthesis, i.e. choosing values for a single parameter, letting others vary randomly, in order to understand how different parameters affect results. Considering $t_{L G} / t_{\text {disp }}$ as the most significant parameter to investigate, we performed the study presented in Figure 9. The 


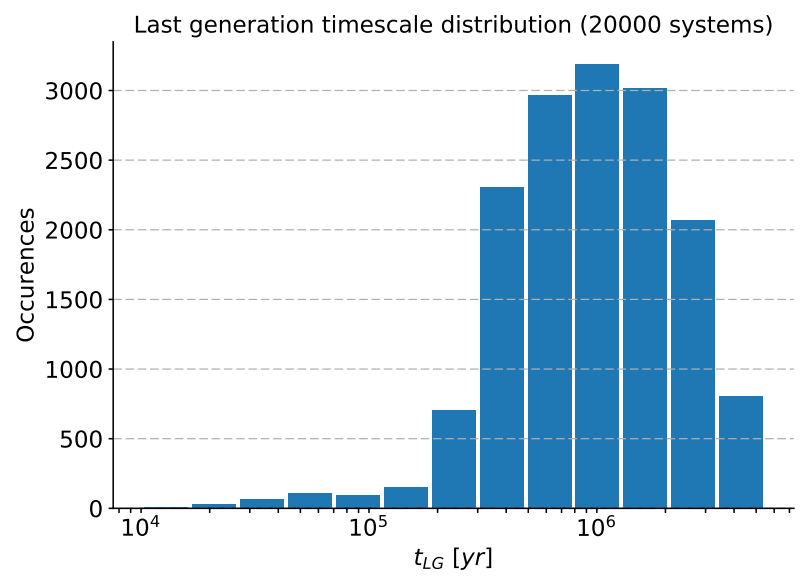

Figure 7. Histogram of all the last generation survival timescales for systems in which at least one satellite survives.

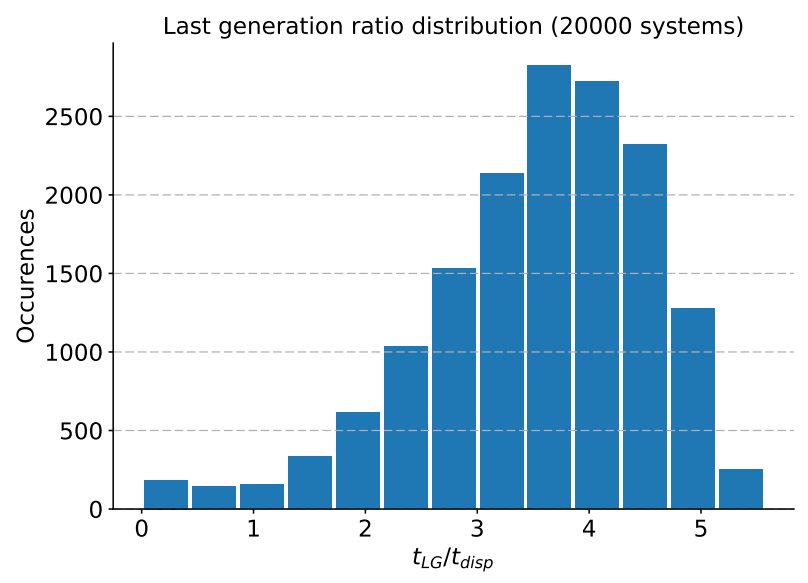

Figure 8. Histogram of all the last generation survival timescales for systems in which at least one satellite survives, divided by $t_{\text {disp }}$ (ratio).

first thing that these plots show is that $t_{L G} / t_{\text {disp }}$, and as a consequence the sequential formation mechanism, is highly dependent on all the parameters we chose in our population synthesis model. For example in the upper panel it is shown that satellites that survives form later, when less dust is available. This is because when the disc is poor of dust it would be difficult to reach streaming instability conditions in the dust trap, then whether the first generation of satellites survives (then we have very short $t_{L G} \mathrm{~s}$ ) or the first generation is lost into the central planet, following generations can not survive.

The second dependence (i.e. $t_{\text {refilling }}$, second panel on Figure 9), shows that the faster the refilling mechanism is, the later the moons form. This is again related to streaming instability conditions in the dust trap because if refilling is efficient the disc would be able to provide enough dust to the dust trap to form a lot of satellites even at later stages. In the lower panel of Figure 9 the dependence on $t_{\text {disp }}$ is plotted. According to this, satellites form later if $t_{\text {disp }}$ is longer. This is not simply linkable to streaming instability conditions as before, but if we assume that, given the same dust-to-gas

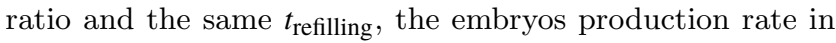
the dust trap is about the same and, if we imagine that $t_{L G}$ is basically given by the lifetime of the disc $\left(\sim 10 t_{\text {disp }}\right)$ minus the production timescale $\Delta t^{1}$, then as a consequence we would have

$\frac{t_{L G}}{t_{\mathrm{disp}}} \simeq 10-\frac{\Delta t}{t_{\mathrm{disp}}}$

i.e. $t_{L G}$ is longer when $t_{\text {disp }}$ is longer. This is obviously an approximation, $\Delta t$ should be (even if slightly) dependent on $t_{\text {disp }}$ as well, but it helps to understand what happens in the model.

\subsection{Formation timescales}

Since we still do not really know on what timescales the Galilean satellites were formed, i.e. how much time a satellite takes to form starting from the formation of its initial embryo, the population synthesis can give a hint about this. Formation timescales have an impact on the structure and composition of the moons, or in reverse, the internal structures of Galilean satellites provide some constraints on the formation timescale. The three inner satellites show a differentiated structure, while Callisto, on the other hand, is not completely differentiated. Differentiation occurs when a satellites (or a body, in general) melts because of the energy received from stellar irradiation, gas interactions, satellitesimals collisions, etc. When this happens heavy elements are allowed to sink toward the center of the satellite, creating different layers. The structure of Callisto gives a first caveat about its evolution, i.e. some believe that its formation timescale could not be shorter than $\sim 10^{5} \mathrm{yr}$ because otherwise collisions and accretion would have transferred energy at a rate high enough to have complete melting (Canup \& Ward 2002; Stevenson et al. 1986). However we have very little knowledge on how the heating/cooling processes worked in the circumplanetary disc that created this moon, nor, where inside the disc Callisto has formed and how its migrated.

In all the simulations it is possible to look at the time needed by any survived satellite to grow to a typical Galilean mass (we chose Europa's mass as a benchmark) and see how these timescales, that we call formation timescale, distribute, leaving out the (few) satellites that do not grow up to Europa's mass. The formation timescale distribution is shown in Figure 10 in which the distribution has a maximum between $10^{4}$ and $10^{5} \mathrm{yr}$ with cases down to $10^{3} \mathrm{yr}$ (about $20 \%$ of the population forms less than $10^{4} y r$ ). This means that satellites can also form very quickly, compared to terrestrial planet formation timescales. This is especially true if the dust-to-gas ratio is high enough in the CPD, the refilling mechanism is efficient and disc dispersion is fast. Previous models, as in Canup \& Ward (2002), predicted quite long timescales, because they did not consider a strong influx from the PPD and, as a consequence, a dust refilling mechanism, instead they just have a low influx rate from the PPD

\footnotetext{
${ }^{1}$ Inverse of production rate.
} 

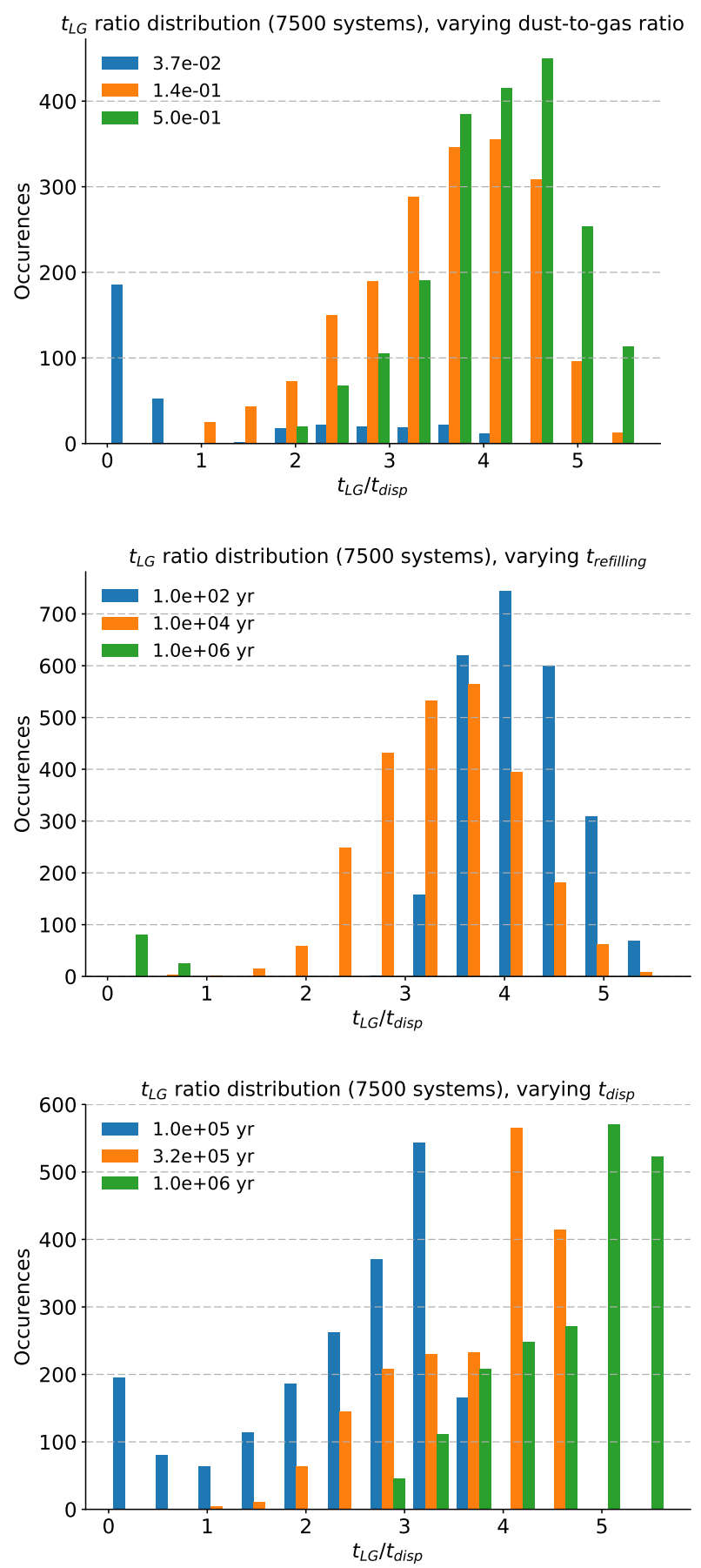

Figure 9. Distributions of $t_{L G} / t_{\text {disp }}$ varying dust-to-gas ratio (top panel), varying the refilling timescale (middle panel), and changing the disc dispersion timescale (lower panel). 2500 simulations were run for each value (7500 simulations in total).

$\left(<10^{-6} M_{J} / y r\right)$ in order to have low temperature and long accretion rates for satellites, to prevent melting and differentiation.

Our results on the formation timescale do not disagree with the $\sim 10^{5} y r$ timescale cited before, because a part of the population is forming on such a long timescale. We have also checked the dependence of the formation timescale on

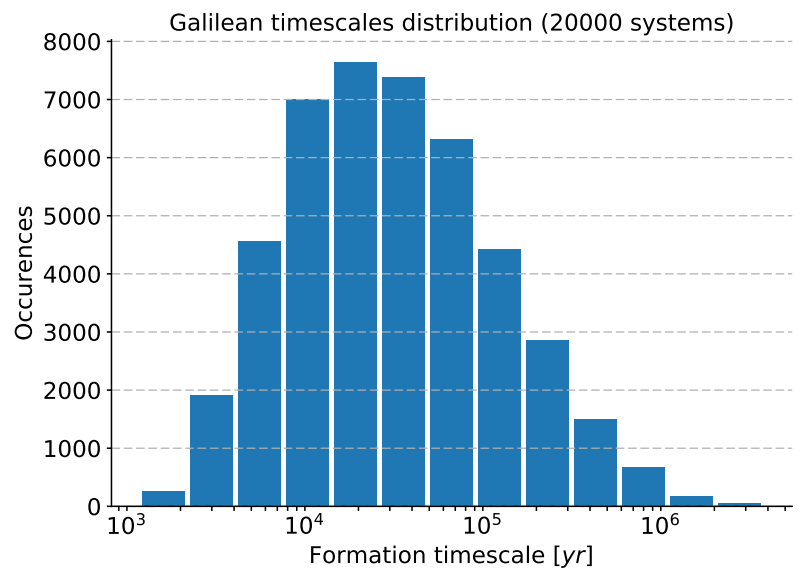

Figure 10. Histogram of all the formation timescales, that distribute with a peak around $2 \times 10^{4} \mathrm{yr}$, with cases in which satellites form even faster than $2-3 \times 10^{3} \mathrm{yr}$.

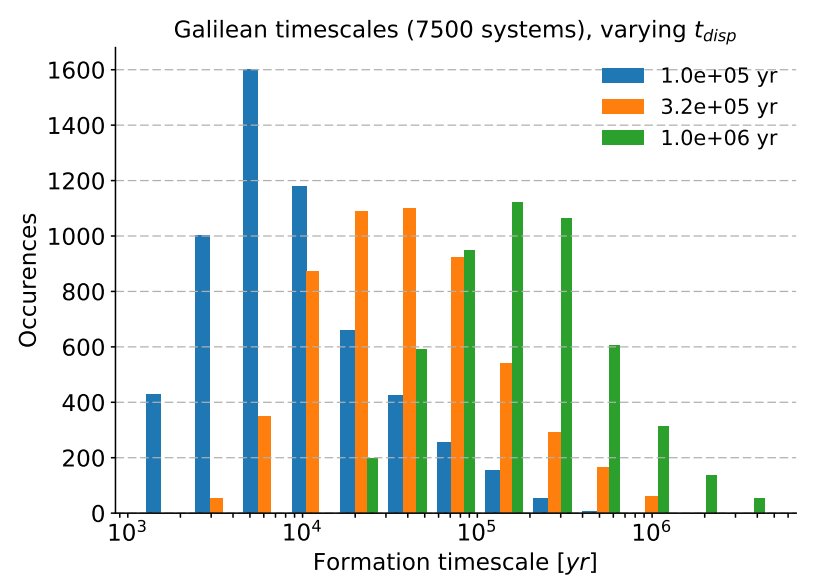

Figure 11. Distribution of the formation timescale while varying the value of $t_{\text {disp }}$, running 2500 simulations for each value (7500 simulations in total).

$t_{\text {refilling, on }} t_{\text {disp }}$, and on the dust-to-gas ratio. Satellites of course form faster and bigger when there is more available dust and/or when refilling is efficient. However, a non-trivial dependence is that on $t_{\text {disp }}$ because it is not possible to link it simply to a general availability of dust or to the efficiency of accretion. The dependence is more related to $t_{L G} / t_{\mathrm{disp}}$, exactly as we described in Section 3.1 with Equation 15. According to this, if $t_{\text {disp }}$ is longer, then $t_{L G} / t_{\text {disp }}$ is longer and the formation process is slower because there is less dust available. This is because the dust density depends exponentially on $t / t_{\text {disp }}$, see Section 2.2 .2 , and the same mechanism apply the other way round. In Figure 11, the dependence of the formation timescale on the disc dispersion timescale is summarized. 


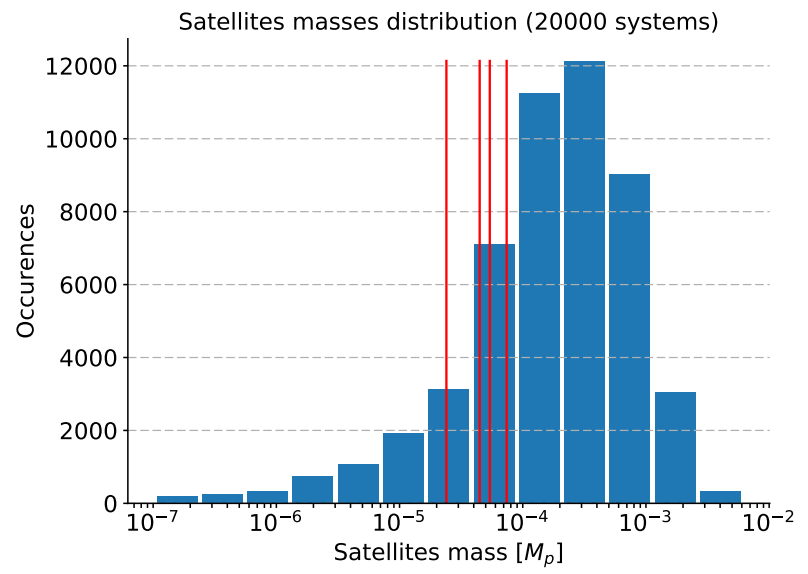

Figure 12. Satellite-mass distribution. The peak can be found between $10^{-4}$ and $10^{-3} M_{p}$, i.e. larger than the Galilean masses, in fact almost at Earth-mass. Red lines indicates the masses of the four Galilean satellites individually.

\subsection{The mass distribution of the satellites}

The satellite-mass distribution is shown in Figure 12, with red vertical lines representing the masses of the four Galilean moons. According to this histogram, the population spreads between $10^{-7} M_{p}$ (i.e. the initial mass of embryos), and $10^{-2} M_{p}$. The peak of the distribution is between $10^{-4}$ and $10^{-3} M_{p}$, which is higher than Galilean masses, often reaching Earth-mass. Only 10\% of the population has a mass similar to Galilean ones.

It was pointed out in Canup \& Ward (2002) that the integrated masses of the moons of Jupiter and Saturn are the same: $M_{i n t}=2 \times 10^{-4} M_{p}$. The authors there discuss the possibility, whether this is coming from physics somehow, whether the CPD-mass is only based on the planetary mass. Recent hydrodynamic simulations have shown, however, that not only the planetary mass sets the CPD-mass, but also the PPD-mass, since the latter continuously feeds the former, hence the more massive PPD will produce a more massive CPD around the same massive planet (Szulágyi 2017). To check those results with population synthesis, in Figure 13 we plotted the histogram of the integrated mass of moons in each individual system of the population. The vertical red line again highlights the Galilean integrated satellite mass: $\left(2 \times 10^{-4} M_{\text {Jupiter }}\right)$. From the Figure it can be concluded that the integrated mass of satellites has a wide distribution, there is no hint for any physical law producing a peak at $M_{i n t}=2 \times 10^{-4} M_{p}$, or at any other particular mass. We therefore conclude, that it is just a coincidence, why the integrated mass of satellites of Jupiter and Saturn are $2 \times 10^{-4} M_{p}$.

We also checked in how many cases, out of the total 20 thousands, we get systems with 3 or 4 satellites with a total mass between $10^{-4} M_{p}$ and $4 \times 10^{-4} M_{p}$, i.e. systems that have masses similar to the Galilean ones. We found that about 4200 systems have such characteristic, i.e. about $21 \%$ of the cases. It is easier to have such systems when the dispersion time of the disc is as long as possible $\left(\rightarrow 10^{6} \mathrm{yr}\right)$ and the refilling timescale is between $10^{4}$ and $10^{5}$ year, while

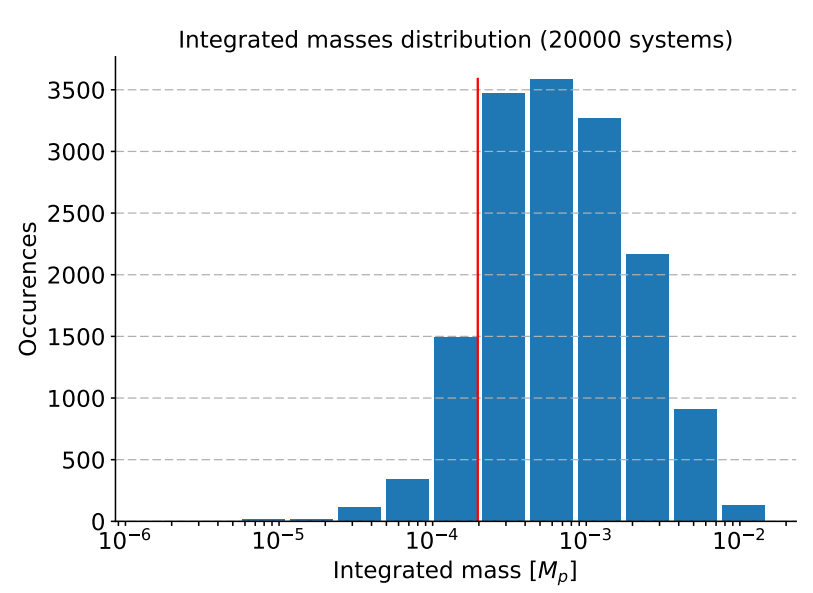

Figure 13. Satellites integrated mass distribution. It has a peak between $10^{-4}$ and $10^{-3} M_{p}$, while the upper limit is about $10^{-1} M_{p}$. The distribution is symmetric. Red line is the Galilean satellites' integrated mass $\left(\simeq 2 \times 10^{-4} M_{p}\right)$.

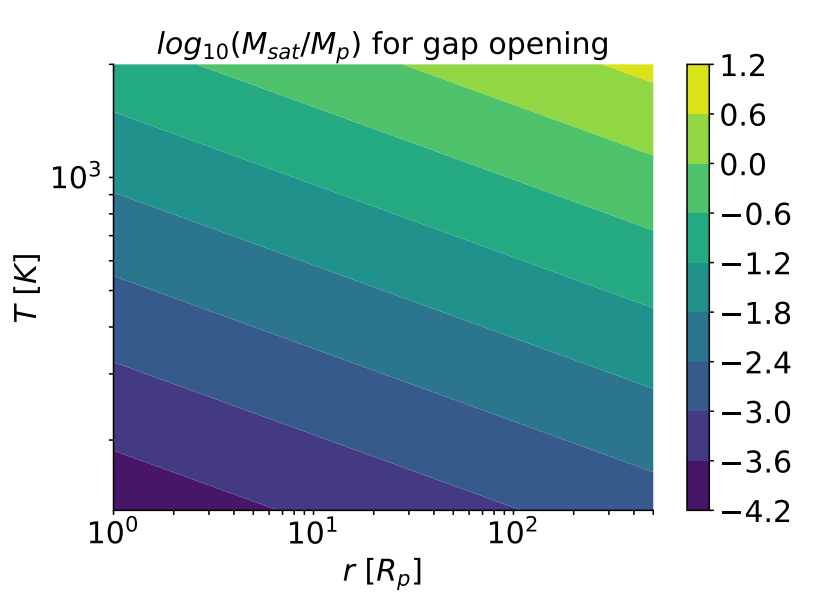

Figure 14. Threshold mass for gap opening $(P<1)$ as a function of $T$ and $r$. In the best configuration, i.e. low temperature close to the planet, a quite a big satellite is still needed to open a gap.

in those cases the value of dust-to-gas ratio can vary in a very wide range (from $5 \%$ to $20 \%$ ).

We also investigated whether moons can open a gap at all in our model. First of all, one can notice that parameter $P$ depends only on the mass of the satellite, the temperature of the CPD, and the position of the satellite in the disc. Hence, it is possible to compute the satellite mass $M_{S}$ that can open a gap, as an analytic function of $r$ and $T$. This way we found that in our model it is very difficult to open a gap at all (Figure 14). In the best case (low temperature close to the central planet) a satellite with $M_{S} \simeq 10^{-4} M_{p}$ is needed, which is a quite high value considering the masses of the Galilean satellites distribute between $10^{-5}$ and $10^{-4} M_{p}$. 


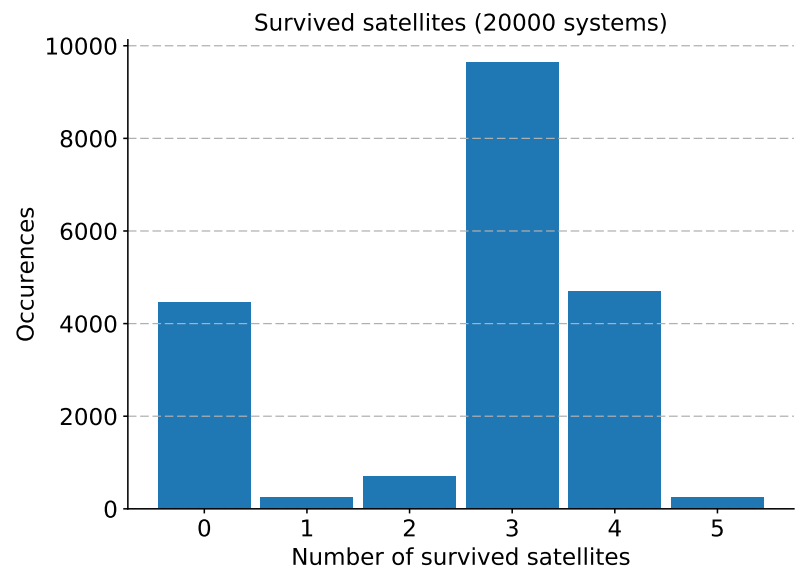

Figure 15. Occurrences of systems with certain numbers of satellites. The peak of the distribution is at 3 , while the upper limit is at 5 . The most peculiar thing is the minimum visible between 1 and 2 .

\subsection{The number of survived satellites}

In Figure 15 we show the satellites that prevail in each one of the 20000 systems after the gaseous CPD (and the PPD) dissipates. In other words these are the moons that exist in the system when the gaseous CPD (and the PPD) dissipates. Without gas, the migration stops, therefore the dynamical evolution of the satellite system has been terminated. The histogram in Figure 15 shows that the most common outcome is a system with 3 satellites. The maximum number of satellites that can be formed in a system is 5 . While 4 is the second most common result, no-survivor case is also frequent. The expectation is that the occurrence rate decrease with increasing amount of moons, however our results show an intriguing minimum at $N=1-2$.

To investigate the reason behind the minimum at 1-2 satellite masses, we used again the second type of population synthesis approach, varying separately the three initial parameters: dust-to-gas ratio, $t_{\text {disp }}$ and $t_{\text {refilling. }}$. We found that changing $t_{\text {disp }}$ does not affect the distribution. This is because the migration timescale, which basically controls the number of coexisting (and then survived) satellites, does not depend on $t_{\text {disp. }}$. This confirms our considerations about $t_{L G}$ in section 3.1. While varying the dust-to-gas ratio, we arrived to the expected result: the more dust produces more satellites, hence more moons survive till the end of the evolution of the disc.

The most extreme difference is found when the refilling mechanism timescale varies (Figure 16). In this case, when refilling is slow, only $0-1$ moons survive, while when refilling is fast, the distribution peaks at around 3 , and this transition occurs between $t_{\text {refilling }}=10^{5}$ and $t_{\text {refilling }}=10^{6}$ years. The shape of the distribution does not change with varying this parameter, the minimum will be always at 2 . With even narrower spacing in the transition region, we revealed that the transition is quite quick and it happens when $t_{\text {refilling }} \simeq$ $1-2 \times 10^{5} y r$

The reason behind this minimum at 2 is the following. In our model, as it was mentioned in Section 2.2.3, embryo

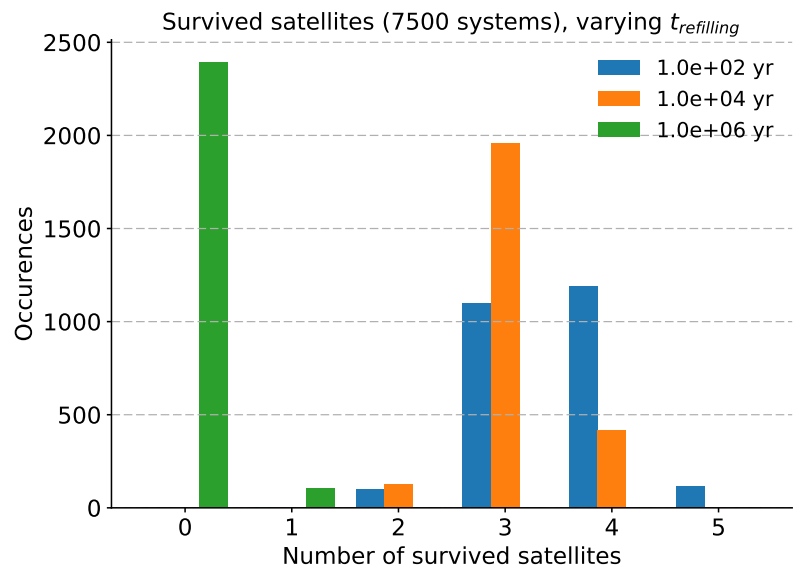

Figure 16. The number of survived satellites when varying only $t_{\text {refilling }}$.

formation is triggered by two conditions: the dust trap has to be out of the previous satellite's feeding zone and the dustto-gas ratio in the dust trap has to be $\geq 1$. The timescales of these two processes strongly depend on $t_{\text {refilling. The first one }}$ because migration depends on the satellites mass, therefore it also depends on the accretion rate. While, in turn, the accretion rate depends on the available dust and refilling, and the dust-to-gas ratio $\geq 1$ depends directly on $t_{\text {refilling. Given }}$ these two conditions, when refilling is fast enough to allow embryos to accrete and to move away from the feeding zone, it is already fast enough to allow dust-to-gas ratio in the dust trap to reach the value 1 at least 3 times in a migration timescale. This means that there would always be at least 3 satellites at the same time, leading to usually 3 survived moons at the end. It is possible to have a few systems with 1 or 2 satellites only when e.g. the disc evolution timescale is fast enough, as shown in Figure 16.

\subsection{Formation temperature}

The composition of the Galilean satellites are very diversified: while Io is completely rocky, the outer three contains some or significant amount of water (Sohl et al. 2002). The water ice can be accreted to the body if it was formed in a disc below the water freezing point, therefore the water content of the Galilean satellites is a strong constraint that the temperature of the forming disc had to be below the water freezing point, $\sim 180 K$ (Lodders 2003). Therefore, we checked the temperature of the disc location where the last survived generation of satellites formed. Because in our model the satelletesimals form in the dust trap, and, most of the dusty material is also generated at this location, we defined a formed moon as icy if the dust trap temperature was below $180 \mathrm{~K}$ in our disc evolution, and as rocky if the temperature was higher than that. We found that $85 \%$ of survived satellites are icy, possibly coexisting with rocky ones.

Like in the previous cases, we also checked how the formation temperature depends on the three parameters individually, which we varied in the population synthesis. The 


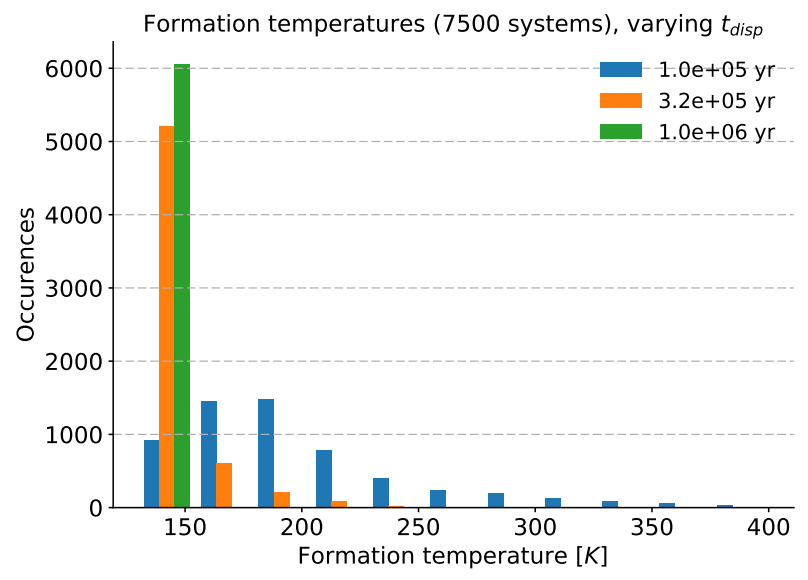

Figure 17. The formation temperatures distribution changes when $t_{\text {disp }}$ is varying. For shorter dispersion timescales the distribution is flatter, with a peak at about $200 \mathrm{~K}$, while for longer dispersion timescales all the distribution concentrate around 130 K.

influence of both the dust-to-gas ratio and the $t_{\text {refilling }}$ is trivial, because in this case they do not have any practical effect on temperatures and almost nothing changes when these two parameters vary. On the other hand, the disc dispersal timescale will affect the temperature evolution of the disc, through the opacities/optical depth. This is clearly visible in Figure 17 where the formation temperature distribution changes shape and moving its peak from about $200 \mathrm{~K}$ to 130 $\mathrm{K}$, as the dispersion timescale is longer.

\section{DISCUSSION}

As it is usual in population synthesis, the choices of the parameters, as well as some assumption on the processes might change the results. In this Section we will discuss this, and describe tests and their results on the model, underlining also the biases that affect this work.

First of all, the disc structure has been modeled starting from the density and temperature profiles in the mid-plane of the disc coming from 3D radiative hydro simulations. All the other features of the disc, such as scale-height, pressure, surface density, sound speed, etc., have been computed from the 1D disc model (Pringle 1981). This is a first approximation that affects some of the CPD features, such as radial velocity profile, opacity and azimuthal velocity, since these quantities strongly depend on, for example, the pressure gradient in the mid-plane, that is computed from the 1D model. Furthermore, for this particular work we used a core accretion formed CPD hydro model. If the CPD forms via disc instability, its properties would be significantly different, see e.g. Shabram \& Boley (2013), Szulágyi et al. (2017a).

Another bias is the disc evolution. For both dispersion and cooling we chose to use self-similar solutions, but, although modeling dispersion of the disc in this way is something common and already used in previous satellite population synthesis works (Ida \& Lin 2008; Miguel \& Ida 2016), a self-similar solution for cooling was a choice taken in order to be consistent with the rest of the semi-analytical description, since it is the first time that CPD-cooling is performed in such a model.

Whether or not there is a magnetospheric cavity between the planet and the disc can affect how many moons are lost in the planet, or whether they could capture into resonances (easily). With no cavity between the planet and the disc, the migration rate of the moons will not be slowed down sufficiently and they will be easily lost in the planet. If there was a disc inner edge, that could hold the inner moons, and, behind, a resonance chain of satellites could pile up (Fujii et al. 2017), like in the case of Super-Earths in PPDs (Ogihara \& Ida 2009). Even in this case, eventually the torque of the newly formed, outer satellites can eventually push the inner moon into the planet. Nevertheless, in this case probably less moons would be lost and more satellites in resonances would be the outcome. In the case of stars, due to the very strong magnetic fields, there is a gap between the surface of the star and the inner PPD. However, giant planets have significantly weaker magnetic fields, Jupiter, for example, has about 7 Gauss today (Bolton et al. 2017). Even though it can be expected, like in the case of stars, giant planets might have stronger magnetic field during their early years than today, there are no studies conducted on the question. There might be a scaling law between the luminosity and the magnetic field as it was pointed out by Christensen et al. (2009), suggesting that forming, luminous planets could have high magnetic field. On the other hand, Owen \& Menou (2016) calculates that Jupiter had to have at least an order of magnitude higher magnetic field than it has today, to induce magnetospheric accretion (and have a cavity between the planet and the disc), and the authors state that it is unlikely that Jupiter ever had such a strong magnetic field. They conclude, that the boundary layer accretion (i.e. when the disc touches the planet surface, like in our hydrodynamic simulations) is a more viable solution. But even if the giant planet has strong magnetic field, in itself this is not a sufficient condition for magnetospheric accretion to start. The gas inside the CPD has to be ionized, otherwise, the neutral gas will not care about the magnetic field and will enter into the cavity region. The ionization fraction of the $\mathrm{CPD}$, on the contrary to the inner PPD, is very low as it was found in several works (Szulágyi \& Mordasini 2017; Fujii et al. 2011, 2014).

Nevertheless, we checked how the results change when a cavity is assumed between the planet and the disc. In this case the first satellite would stop at the edge of the disc. The following satellite would then approach the first one and it would possibly be caught in a $2: 1$ resonant configuration. Whether or not this capture happens can be inferred from analytical conditions, e.g. in Ogihara et al. (2010). In their work they found that, in case of a sharp disc edge and using the type I migration formula by D'Angelo \& Lubow (2010) for its simplicity (we show below that changing the type I migration formula does not change our results significantly), up to 3 satellites would be locked in a resonant configuration when $t_{e} / t_{a}<1.7 \times 10^{-3}$, where $t_{e}$ is the eccentricity damping timescale and $t_{a}$ is the type I migration timescale. In our case this criterion implies a condition on the aspect ratio of the disc at the inner edge, i.e. $h / r<0.024$. Using the definition of $h$ in a 1D disc model (Pringle 1981) one finds 
the condition

$\frac{T}{[K]} \frac{r_{\text {cavity }}}{R_{p}} \leq 210$

where $T$ is the temperature at the inner edge of the disc. This means that if we want to pile satellites up starting from the position of Io $\left(\simeq 6 R_{p}\right)$ we need to have a temperature of about $35 K$, that is unphysical, due to the background temperature at Jupiter's location is about $130 \mathrm{~K}$.

Even if building a resonant structure is not possible in our model, we checked how the final results change when a cavity (as big as $2.5 R_{p}$ or $5 R_{p}$ ) is introduced. In this case we considered that satellites stop their migration due to gas interaction when reaching the inner edge of the disc, but they do interact dynamically still with other satellites. This means they still can be lost into the planet. The interaction between satellites has been modeled following the approach of Ida \& Lin (2010), i.e. considering that satellites tend to enlarge their orbital distance $\Delta a$ at each encounter. As expected, we found that we have more surviving satellites (their mean number grows from 2.5 in the case without a cavity to 3.8 and 4.5 respectively, when the two different cavities are introduced) and as a consequence the integrated final moon mass grows from a median value of $6 \times 10^{-4} M_{p}$ to $8 \times 10^{-4} M_{p}$ and $12 \times 10^{-4} M_{p}$, respectively, while the mean mass of single satellites does not change significantly.

In this work we also assumed that streaming instability forms the seeds of the moons. The more traditional approaches would work with lower dust-to-gas ratios, but would provide much longer formation timescales and would be difficult to jump over known the size-barriers famous for these models. Given that our hydrodynamical simulations have showed that dust traps appear in CPDs, it was a natural assumption that streaming instability can operate. Another mechanism, that could have provided the seeds is the capturing of planetesimals from the PPD (D'Angelo \& Podolak 2015; Tanigawa et al. 2014). Given that we found that the CPD is an efficient satelletesimal factory, we believe that there is no need for planetesimal capturing to form the moons there.

Regarding testing the initial parameters, we first checked the effect of initial embryo mass and a different Type I migration formula. In the latter, instead of the Paardekooper-formula (Paardekooper et al. 2011) we tested the $b_{\boldsymbol{I}}$ coefficient from D'Angelo \& Lubow (2010) and Dittkrist et al. (2014). Our finding is that the distribution of the population does not change much, the difference is within the change that is caused by random variations.

In comparison to the previous satellite population synthesis work by Miguel \& Ida (2016), our results are somewhat different. While the other authors started with a Minimum Mass Sub-solar Nebula that is created by the current position and composition of Galilean moons, we used real hydrodynamic simulations on the circumplanetary disc as an initial gas and dust disc. Unlike them, we also take into account the disc evolution both in dust density and in temperature, and we do not consider a cavity between the planet and the disc. They find that in the case of long disc lifetimes, the survived satellites are less numerous and has lower masses than in our case, since the biggest ones have enough time to migrate and be lost into the central planet. The difference comes from the different dust-to-gas ratios, different disc initial parameters, and the assumption which process generates the seeds of satellites, but also from the fact that they do not have any dust supply in the disc while accretion on protosatellites creates gaps in the dust profile. As a consequence their protosatellites have less available dust to grow to larger sizes.

\section{CONCLUSION}

In this work we investigated the formation and the evolution of the Galilean satellites in a circumplanetary disc around a Jupiter-like planet. We used a population synthesis approach involving 20000 systems, using the initial conditions (disc density and temperature profiles) from a 3D radiative simulation of Szulágyi (2017), including the continuous feeding of gas and $<\mathrm{mm}$ sized dust from the feeding of the protoplanetary disc (Szulágyi et al. 2014). In the population synthesis, we accounted for the disc evolution and used a dust density profile from a realistic dust coagulation model of Drazkowska 85 Szulagyi (in prep). Furthermore, in our model the seeds of the moons form via streaming instability in a dust trap, whose location is around $80 R_{J u p}$ based on the vertical velocity profiles of the hydrodynamic simulation. The satellitesimals then migrate, accrete, are captured in resonances and are often lost in the planet.

Nevertheless, we found that due to the dust trap, and the continuous influx of dust from the circumstellar disc, massive satellites are forming (the distribution peaks above the Galilean mass at $\left.\simeq 3 \times 10^{-4} M_{J} \simeq M_{\text {Earth }}\right)$. Due to their high masses, they quickly migrate into the planet via Type I migration, because in most of the cases the gap opening criterion is not satisfied, the migration cannot enter the Type II regime. This means that the satellites form in sequence, and many are lost into the central planet polluting its envelope with metals. Our results show that the moons are forming fast, often within $10^{4}$ years (20\% of the population), which is mainly due to the short orbital timescales of the circumplanetary disc. Indeed the CPD completes several orders of magnitude more revolutions around the planet than the protoplanetary disc material can do around the star at the location of Jupiter. Due to the short formation time, the satellites can form very late, about 30\% after 4 dispersion timescales, i.e. when the disc has $\sim 2 \%$ of the initial mass. Due to our model included disc evolution, the CPD cooled off during this time, allowing to form icy moons, when the dust trap temperature dropped below $180 \mathrm{~K}$, i.e. the water freezing point. We found out that about $85 \%$ of the survived moons could contain water (ice). The production of moonlets and the migration rate provided such a situation, when the number of survived moons peaked around 3 , but often no moons survived at all.

The lost satellites bring on average 0.3 Earth-masses into the giant planet's envelope, polluting it with metals, that can contribute to the abudance of heavy elements in Jupiter's envelope. The high mass satellites we found in our population synthesis can be an encouraging result for the future surveys of exomoons, because even with the current instrumentation, an Earth-mass planet around a Jupiter analog can be detected if the planet is orbiting relatively close to its star (Kipping 2009). 


\section{ACKNOWLEDGMENTS}

We are thankful for the useful discussions with Yann Alibert, Christoph Mordasini and Clement Baruteau. This work has been in part carried out within the frame of the National Centre for Competence in Research "PlanetS" supported by the Swiss National Science Foundation. J. Sz. acknowledges the support from the ETH Post-doctoral Fellowship from the Swiss Federal Institute of Technology (ETH Zürich) and the Swiss National Science Foundation (SNSF) Ambizione grant PZ00P2_174115. Computations have been done on the "Mönch" machine hosted at the Swiss National Computational Centre.

\section{REFERENCES}

Alibert, Y., Mousis, O., \& Benz, W. 2005, A\&A, 439, 1205

Anderson, J. D., Jacobson, R. A., Lau, E., Sjogren, W. L., Schubert, G., \& Moore, W. B., 1996, Nature, 384, 541

Anderson, J. D., Jacobson, R. A., Lau, E., Sjogren, W. L., Schubert, G., \& Moore, W. B., 1998, Science, 5369, 1573

Anderson, J. D., Jacobson, R. A., Lau, E., Moore, W. B., \& Schubert, G., 2001, Icarus, 153, 157

Ayliffe, B. A. \& Bate, M. R., 2009, Mon. Not. R. Astron. Soc. 397, 657-665

Bell, K. R., \& Lin, D. N. C. 1994, ApJ, 427, 987

Benz, W., Ida, S., Alibert, Y., Lin, D., \& Mordasini, C., 2014, Protostars and Planets, VI

Bolton, S. J., Adriani, A., Adumitroaie, V., et al., 2017, Science, $356,821-825$

Boss, A. P., 1997, Science, 276, 5320, 1836-1839

Boulanger, F., Cox, P., \& Jones, A. P., 2000, Infrared space astronomy, today and tomorrow, 70, 251

Canup, R. M. \& Ward, W. R., 2002, The Astronomical Journal, 124, 3404-3423

Canup, R. M. \& Ward, W. R., 2006, Nature, 441, 834-839

Canup, R. M. \& Ward, W. R., 2009, Origin of Europa and the Galilean Satellites, 59

Cameron, A. G. W., 1978, Moon and Planets, 18, 5-40

Commerçon, B., Teyssier, R., Audit, E., Hennebelle, P., \& Chabrier, G. 2011, A\&A, 529, A35

Crida, A. \& Morbidelli, A., 2014, Mon. Not. R. Astron. Soc. 377, $1324-1336$

Christensen, U. R., Holzwarth, V., \& Reiners, A. 2009, Nature, 457,167

D'Angelo, G., Henning, T., \& Kley, W. 2002, A\&A, 385, 647

D'Angelo, G. \& Lubow, S. H., 2010, ApJ, 724, 730-747

D'Angelo, G., \& Podolak, M. 2015, ApJ, 806, 203

de Val-Borro, M., Edgar, R. G., Artymowicz, P., et al. 2006, MNRAS, 370, 529

Dittkrist, K.-M., Mordasini, C., Klahr, H., Alibert, Y., \& Henning, T., 2014, Astronomy \& Astrophysics, 567, A121

Drazkowska, J., \& Alibert, Y., 2017, A\&A 608, A92

Duffell, P. C., 2011, ApJLetters, 807, L11

Durisen, R. H., Boss, A. P., Mayer, L., Nelson, A. F., \& Quinn, T., 2007, Protostars and Planets V, 607-622

Ercolano, B., Mayr, D., Owen, J. E., Rosotti, G., \& Manara, C. F., 2014, MNRAS, 439, 256-263

Estrada, P. R., Mosqueira, I., Lissauer, J. J., DâĂŹAngelo, G., \& Cruikshank, D. P., 2009, in Europa, ed. R. T. Pappalardo, W. B. McKinnon, \& K. Khurana (Tucson, AZ: Univ. Arizona Press), 27

Fedele, D., van den Ancker, M. E., Henning, T., Jayawardhana, R., \& Oliveira J. M., 2010, A\&A 510, A72

Fujii, Y. I., Okuzumi, S., \& Inuzuka, S., 2011, American Astronomical Society, ESS meeting 2
Fujii, Y. I., Okuzumi, S., Tanigawa, T., \& Inuzuka, S., 2014, The Astronomical Journal, 785, Issue 2

Fujii, Y. I., Kobayashi, H., Takahashi, S. Z., \& Gressel, O., 2017, The Astronomical Journal, 153, Issue 4

Fung, J., \& Chiang, E. 2016, ApJ, 832, 105

Galvagni, M. \& Mayer, L., 2012, Mon Not R Astron Soc, 427, $1725-1740$

Greenberg, R., Bottke, W. F., Carusi, A., \& Valsecchi, G. B., 1991, Icarus 94, 98-111

Gressel, O., Nelson, R. P., Turner, N. J., \& Ziegler, U., 2013, ApJ, 779,1

Hayashi, C., 1981, Progress of Theoretical Physics Supplement, 70,35

Ida, S. \& Lin, D. N. C., 2008, ApJ, 673, 487-501

Ida, S. \& Lin, D. N. C., 2010, ApJ, 719, 810-830

Jewitt, D. \& Hanghighipur, N., 2007, A\&A, 45, 1, 261-295

Kipping, D. M., 2009, Mon Not R Astron Soc, 392, 181-189

Kley, W., 1989, A\&A, 208, 98

Lodders, K. 2003, ApJ, 591, 1220-1247

Lubow, S. H., Seibert, M., \& Artymowicz, P. ,1999, ApJ, 526, 1001

Mamajek, E. E. 2009, AIP Conference Proceedings, 1158, 3-10

Miguel, Y. \& Ida, S., 2016, Icarus, 266, 1-14

Mosqueira, I. \& Estrada, P. R., 2003, Icarus, 163, 198-231

Ogihara, M. \& Ida, S., 2009, ApJ, 699, 824-838

Ogihara, M., Duncan, M. J., \& Ida, S., 2010, ApJ, 721, 1184-1192

Owen, J. E., \& Menou, K. 2016, ApJ, 819, L14

Papaloizou, J. C. B. \& Nelson, R. P., 2005, A\&A, 433, 247-265

Paardekooper, S.-J., Baruteau, C., \& Kley, W., 2011, Mon. Not. R. Astron. Soc., 410, 293

Pollack, J. B., \& Consolmagno, G., 1984, in Saturn, ed. T. Gehrels \& M. S. Matthews (Tucson: Univ. Arizona Press), 811

Pollack, J. B., 1996, Icarus, 124, 1, 62-85

Pringle, J. E., 1981, Annual review of astronomy and astrophysics, $19,137-162$

Sasaki, T., Stewart, G. R., \& Ida, S., 2010, ApJ, 714, 1052-1064

Shabram, M. \& Boley, A. C., 2013, ApJ, 767, 63

Showman, A. P. \& Malhotra, R., 1999, Science, 296, 77-84

Sohl, F., Spohn, T., Breuer, D., \& Nagel, K., 2002, Icarus, 157, 104

Stevenson, D. J., Harris, A. W., \& Lunine, J. I., 1986, Satellites (A87-23307 09-91), University of Arizona Press, 39-88

Syer, D. \& Clarke, C. J., 1995, Mon. Not. R. Astron. Soc. 277, 758-766

Szulágyi, J., Morbidelli, A., Crida, A., \& Masset, J., 2014, ApJ, 782,65

Szulágyi, J., Masset, F., Lega, E., Crida, A., Morbidelli, A., \& Guillot, T., 2016, Mon. Not. R. Astron. Soc., 460, 2853-2861

Szulágyi, J., Mayer, L., Quinn, T., 2017, Mon. Not. R. Astron. Soc., 464, 3158-3168

Szulágyi, J., \& Mordasini, C. 2017, MNRAS, 465, L64

Szulágyi, J. 2017, ApJ, 842, 103

Szulágyi, J., van der Plas, G., Meyer, M. R., Pohl, A., Quanz, S. P., Mayer, L., Daemgen, S., \& Tamburello, V., 2017, accepted for publication at Mon Not R Astron Soc

Tanaka, H., Takeuchi, T., \& Ward, W. R., ApJ, 565, 1257

Tanigawa, T., Ohtsuki, \& Machida, M. N., 2012, European Planetary Science Congress, 7, 610

Tanigawa, T., Maruta, A., \& Machida, M. N. 2014, ApJ, 784, 109

Ward, W. R. \& Canup, R. M., 2010, The Astronomical Journal, 140, 1168-1193

Wilkins, D. R. \& Clarke, C. J., 2012, Monthly Notices of the Royal Astronomical Society, 419, 4, 3368-3377

Youdin, A. N. \& Goodman, J., 2005, ApJ, 620, 459-469

Zhu, Z., Hartmann, L., \& Gammie, C., 2009, ApJ, 694, 1045-1055 